\title{
Difficult DJ stent Removal - Our Experience and Evolution of Institutional Protocol for Management of Stuck DJ stent
}

\author{
Dr. M. Nazar ${ }^{1}$ M Dr Vikram Nimboor ${ }^{2}$ Dr Tony $T^{3}$ Dr Aby Madan ${ }^{4}$ \\ Dr Shankar Ram HS ${ }^{5}$ Dr Rahul Raveendran ${ }^{6}$ Dr Jithender ${ }^{7}$ Dr Nityanand ${ }^{8}$ \\ 1. Additional Professor. Department of Urology, Government, Kerala. INDIA \\ 2. Resident. Department of Urology, TD Medical College, Alappuzha Kerala. INDIA \\ 3. Assistant professor. Department of Urology, Government, Kerala. INDIA \\ 4. Assistant Professor. Department of Urology, Government, Kerala. INDIA \\ ${ }^{5678}$ Resident. Department of Urology, TD Medical College, Alappuzha Kerala. INDIA \\ Email For correspondence: drmnazaruro@gmail.com
}

\begin{abstract}
Introduction :Stent removal is a routinely done urological procedure and at times we encounter difficulty in stent removals. It is important to follow careful plan of action to avoid complication.

Our objective is to make a simple stepwise protocol for their management and to present our series of patients with difficult stent removals and various management options that we had used. Methods: We collected data of all the patients who had come to us for stent removal from 2013 to 2015 . Total of 596 stent removals were performed and 31 patients had difficult stent removal in patients who had undergone stenting after URS, ESWL, Infected hydronephrosis and others.

Results: Using the simple stepwise protocol we could manage all our patients without any complications. All Patients underwent $3 D$ NCCT KUB reconstruction to delineate the encrustations and were managed accordingly as per the location in the protocol. Upper tract encrustations received ESWL, lower tract received cystolitholapaxy/tripsy, and ureteric encrustations were managed by URSL. PCNL was done for failed ESWL (Large hard encrustations, PCN was placed).

Conclusions: D-J stents have been widely used since many years and this has corresponded to the increase in possible complications including stent migration, encrustation, stone formation, and stent fracture. We have come up with a management protocol that is simple to follow and easy to execute and helps to avoid disastrous complications.
\end{abstract}

Key words: Difficult DJ stent Removal,Encrustations ,ESWL, URSL ,Cystolithotripsy. PCNL, dDJSR (difficult DJ stent Removal)

\section{Introduction}

Stent removal is a routine urological procedure and at times we encounter difficulty in DJ stent removals. Despite proper follow up and timely scheduled stent removal we still encounter difficulty in stent removal. Encrustations are seen mostly in the coils of the DJ stent.An incomplete clearance of the stone, stents in recurrent stone formers, stents in patients with metabolic abnormalities, presence of factors for infection increases the chances of encrustations .Apart from these ,there are rarely stent fracture and stent migration, knotting with or without encrustation that cause difficulty in stent removal. Every institution should have its protocol for the management of difficult stent removal. ${ }^{(1-2)}$

\section{II .Methods}

We collected data from our stent register - from 2013 to 2015 .Total of 596 stent removals were performed and there were 31 difficult stent removals. A stepwise protocol is devised in our institution which is applied and followed.

Figure 1.Protocol for difficult Stent Removal . 


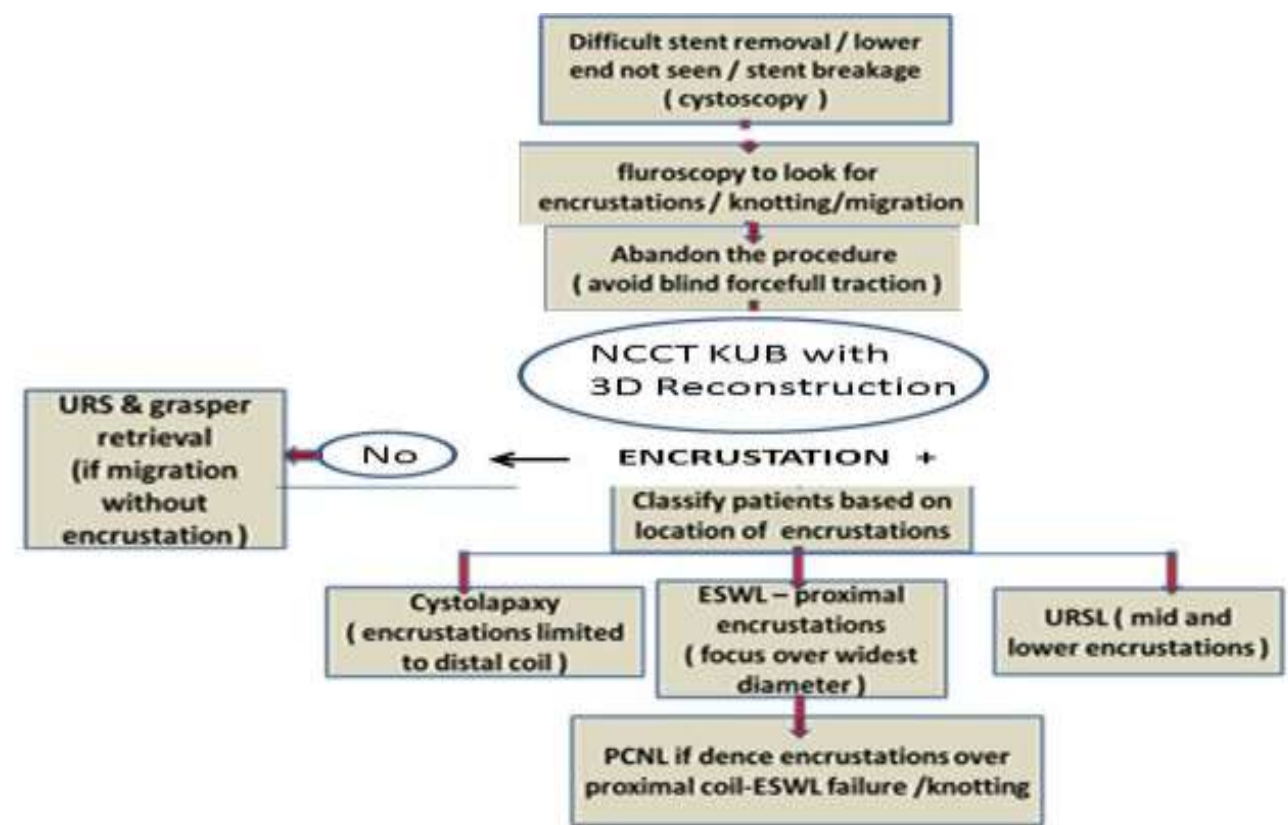

Figure 2 . 3D Reconstruction in different views to delineate encrustations
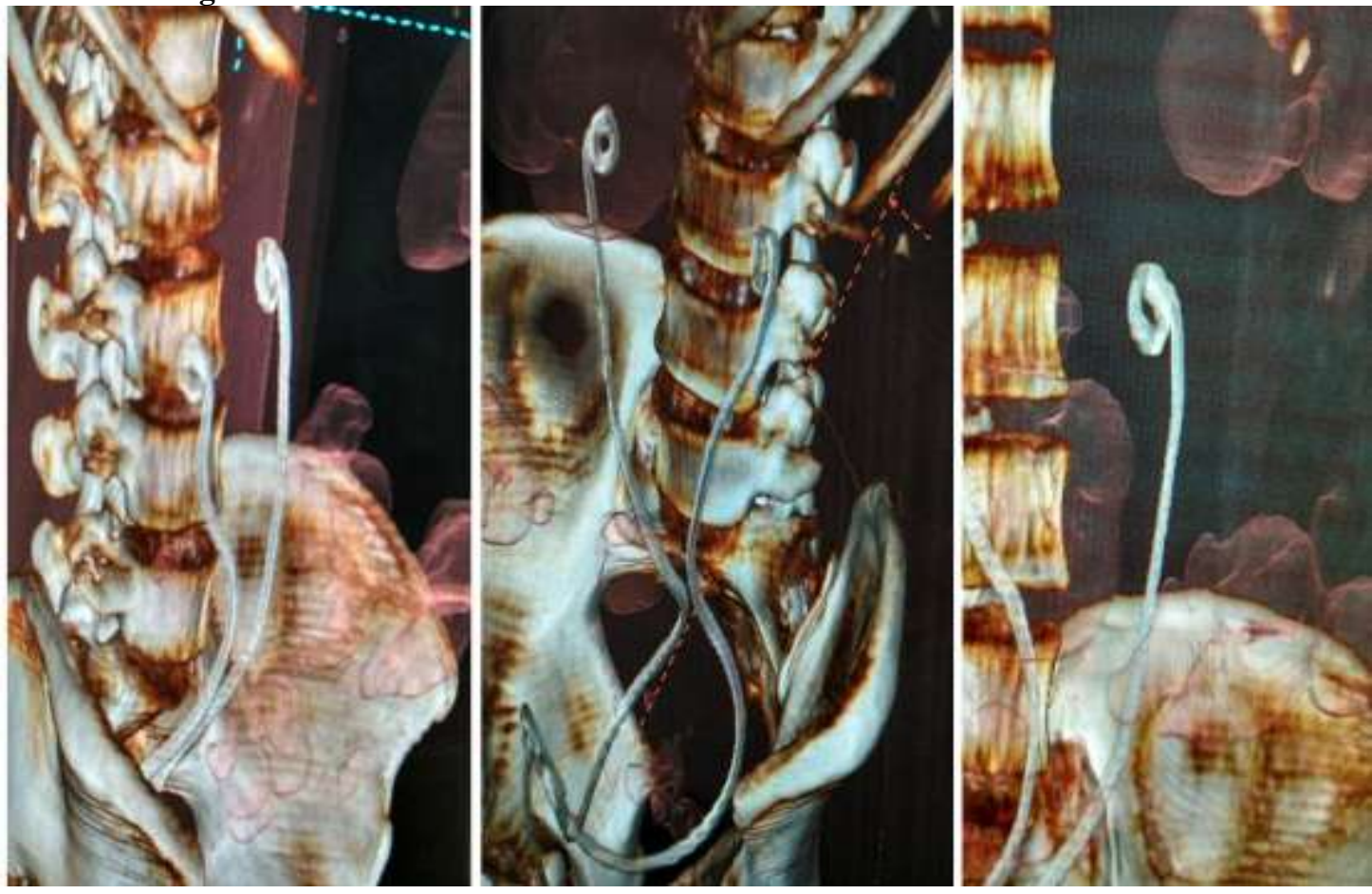

\section{Results}

III.

There were 31 patients with difficult stent remove who underwent at least a second procedure for stent removal .The reason for placement of stent are given in figure 2 below. 70 per cent of difficult stent removal had significant upper coil and 45 per cent had significant lower coil encrustations though encrustations were present through out and nearly half shared significant encrustations at both the ends .Majority of the significant encrustations when present at the lower coil had significant encrustations in the upper end .Ureteric encrustations were smaller and manageable with URSL. Smaller encrustations in the lower coil could be removed with minimal manipulations without any additional procedures in the first attempt itself under fluoroscopy and hence not included in difficult stent removals.. 


\begin{tabular}{|l|l|}
\hline Stent removal following & $\begin{array}{l}\text { No. of } \\
\text { patients }\end{array}$ \\
\hline $\begin{array}{l}\text { Post ESWL (scheduled) } \\
\text { Post URS (scheduled) }\end{array}$ & 9 \\
$\begin{array}{l}\text { Post URS (Delayed 12-24 } \\
\text { months) }\end{array}$ & 4 \\
Obstructive uro sepsis & 5 \\
\hline Neglected stent (3.5 yrs - URS) & 1 \\
\hline
\end{tabular}

$\begin{array}{lc}\text { ESWL + URS } & 11 \\ \text { ESWL + URS + PCNL } & 1 \\ \text { PCN+PCNL } & 1 \\ \text { URS } & 3 \\ \text { CLP/CLT } & 2 \\ \text { URS +CLT } & 4 \\ \text { ESWL + CLT } & 4 \\ \text { ESWL + URS } & 2 \\ \text { ESWL + URS + CLT } & 3\end{array}$

Management of Encrustation as per protocol . Number of procedures done in 31 patients .

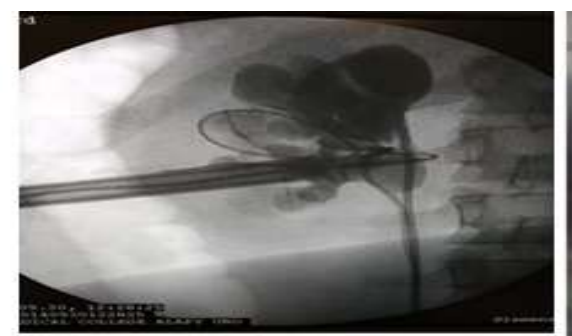

PCNL for dense encrustations and knotting - over proximal stent coil
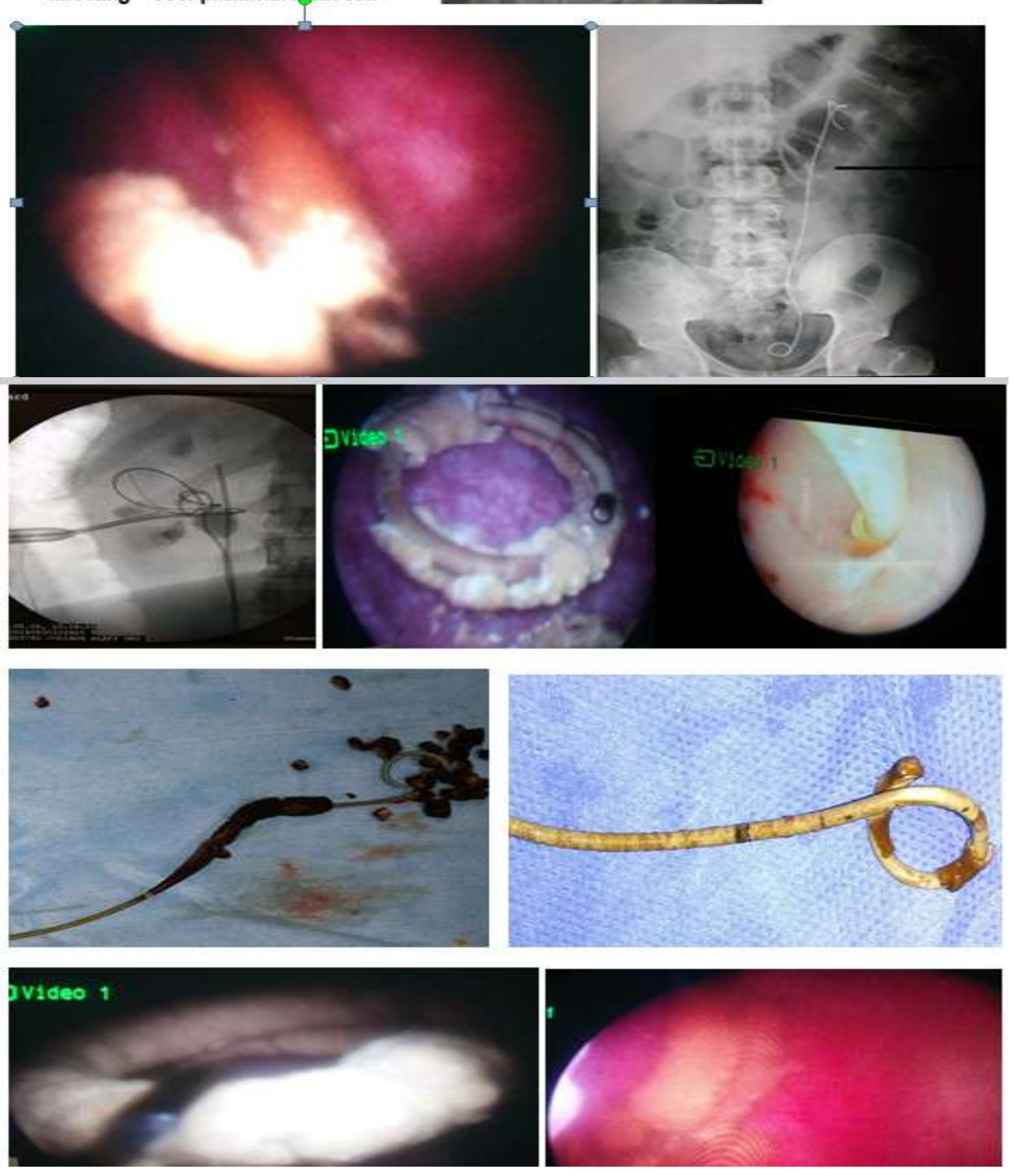

DOI: $10.9790 / 0853-1603022124$

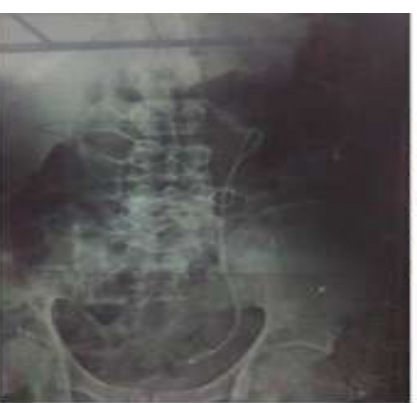

Knotting with

dense

encrustations

over upper stent

coil
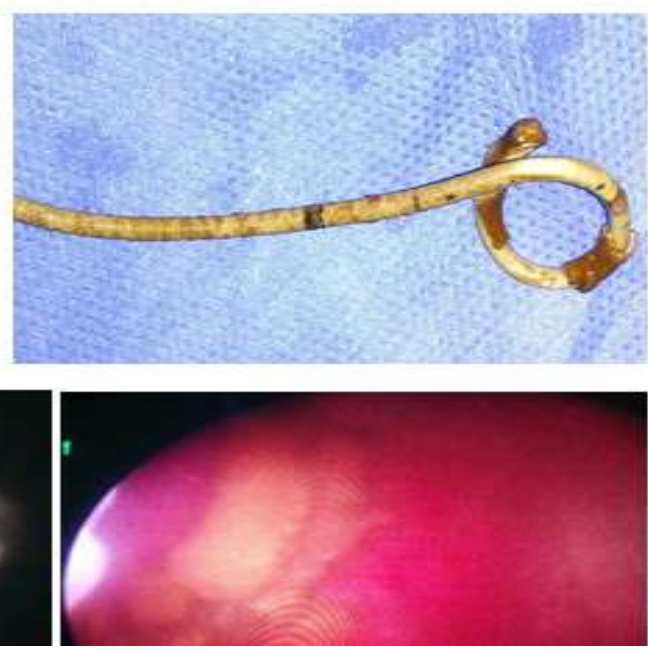

Www.iosrjournals.org 


\section{Discussion}

Encrustations which are early have lower HU and can be missed in X ray KUB and Fluroscopy. But Indirectly can be made out when fluoroscopy is immediately supplemented while ascertaining difficult DJ stent Removal .The upper coil do not open up and descend as such when DJ stent is being removed by cystoscopy.A simultaneous RGP(not necessary) will show kinking down of Pelviureteric junction by the unopened upper coil of the DJ stent .As soon as this finding is encountered the procedure is immediately abandoned. Any difficulty in the form of unusual resistance we proceed for NCCT - KUB with 3D Reconstruction that delineates all the encrustations associated with DJ stent and also residual fragments/ encrustations if any along the entire tract of the stent. The console images are examined by urologists in detail and in all orientations. These are also correlated with the radio opaque zones along the stent in X rays .Further management is planned depending the site ,size ,location(s),extent of the encrustation ,HU of encrustation (low if radiolucent) . Contrast studies are performed further if required as and when the scenario demands .

\section{Encrustations in Upper ureter near PUJ, ,Part of Upper Ureter,Pelvis and Calyx}

2 cycles of ESWL over one week apart with 2000 shocks $60 \mathrm{hz}$, graded 12- $15 \mathrm{kv}$ over the coil /encrusted segments as identified - are given for all upper tract encrustations.A repeat imaging is done after 6 weeks (USG KUB screening or NCCT KUB if indicated ) in patients in whom DJ stent removal was possible after only ESWL without upper tract instrumentation. Cases of tract obstruction with altered RFT and / sepsis are managed with PCN with or without HD till patient is stabilised .PCNL is done in case of hard (High HU > 1300 - large radio opaque encrustation), failed ESWL ,Extensive knotting and dense entrapment, if already nephrostomy is present, failed URSL.

Part of Upper ureter, mid , and lower ureter. Contrast studies in form of urogram /RGP is supplemented as and when necessary. Kinks, redundant and impliable segments of ureter can be made out. Whenever any undue resistance is felt at stent withdrawal (always under C arm at time of diagnosis / while intraoperative management) a simultaneous RGP might show these findings. By passing a $6 \mathrm{~F}$ Ureteroscope and negotiating along the stent without telescoping of the stent and ureter the encrustations are meticulously fragmented in a stepwise manner till the pelvis. Whenever possible after clearing the encrustations in the lower coil the distal end of the stent could be brought outside the meatus especially in female patients and can be fixed.This avoids migration, coiling of the stent in the lower ureter before/ while proceeding higher up in to the ureteric encrustations giving adequate space for the ureteroscope to reach $u$ to the pelvis . Lithotripsy (Pneumatic or Laser) is done to ensure complete fragmentation.

Lower coil /Bladder encrustation- Exclusive bladder encrustations are managed by Cystolithotripsy/Cystolithopaxy . All the management procedures are done under fluoroscopy under Spinal or General Anesthesia.After the DJ stent removal a diagnostic uretersoscopy with semirigid/flexible is done to look for residual encrustation only if necessary / possible in the same sitting at the end. Graspers and baskets were used to down phase the stent whenever required at management.. Multimodal approach/multistage approach is used depending on scenario.

Apart from the patient factors ,non compliant follow up ,we understand that Quality of the stent also matters depending on the brand and batch of which some are prone for rapid onset of encrustation even in non recurrent stone formers and those who had stent for reasons other than stone disease .Setting up of standards for stent manufacturers and quality control measures can help in avoiding non patient related difficult stent removals. Also one should alert the susceptible patients for early stent removal especially with long duration stents when are placed. Selection of appropriate DJ stent length also avoids coiling, knotting and migration .Polyurethane stents compared to Silicone stents have less chance of migration ${ }^{3}$. Some times patients return early uncomfortable with symptoms of increasing pain, hematuria, fever, chills, rigors , passage of encrustations, recurrent UTI and even Urosepsis. The possibility of these symptoms of DJ stent origin has to be kept in mind and approached.

The stasis of Urine in bladder and Pelvis relative to the peristaltic ureter , presence of reflux might alter the deposition rates of encrustations over the DJ stent ${ }^{4}$.An encrustation at upper coil cannot cross the PUJ and at the lower coil cannot pass the bladder outlet to reach urethra and can injure them .Unless otherwise proved an encrustation seen during cystoscopy at DJSR in the lower end had a high probability of encrustations elsewhere in the DJ stent and the procedure should be immediately abandoned after single failed attempt under fluoroscopy.

\section{Conclusion}

Our stepwise protocol is effective and successful in the management of Difficult stent Removal.Following this stepwise protocol during difficult stent removals we were able to avoid complications like bleeding, mucosal injury and rarely PUJ avulsion. Safety is the more important at this juncture as one complication shouldn't lead to another.

\section{References}

[1]. Successful Treatment of Stent Knot in the Proximal Ureter Using Ureteroscopy \& Holmium Laser. Masters M. Department of Urology, University of Arkansas, AR 72205, USA Volume 2011, Article ID 502191, doi:10.1155/2011/50211

[2]. Knotted stents: Case report and outcome analysis - Min Su Kim, Department of Urology, Seoul Medical Center, Seoul Korean J Urol 2015;56:405408

[3]. Youness A,Khallouk A ,Jamal M, Hassan M . Risk Factor Analysis and Management of Ureteral Double J stent Complications . Reviews in Urology . 2010.Vol 12 .no 2/3.;147-150.

[4]. .Y Dakkak,A Janane, Ould Ismail T, Ghadouane M ,Ameur A ,Abbar M. . Management of encrusted ureteral stents .African Journa of Urology .2012;18:131-134 